\title{
Effect of TRAIL in combination with DDP on the expression of MDR1 gene in gastric cancer cells
}

\author{
Yu-Fang Cui, Long-Shan Yu, Hui-Qun Wang, Ya-Wen Gou, Qiao-Ming Wang, Kai-Guang Zhang \\ Department of Gastroenterology, The Affiliated Provincial Hospital of Anhui Medical University, Hefei City, China
}

Prz Gastroenterol 2014; 9 (4): 214-219

DOI: $10.5114 / p g .2014 .45103$

Key words: gastric carcinoma, TRAIL, MDR1, P-glycoprotein.

Address for correspondence: Prof. Kai-Guang Zhang MD, PhD, Department of Gastroenterology, The Affiliated Provincial Hospital of Anhui Medical University, 81 Meishan Road, 230001 Hefei City, Anhui Province, China, phone: +8613855170097, e-mail: zkg@medmail.com.cn

\begin{abstract}
Introduction: Gastric cancer is one of the most common malignant tumor, and gastric cancer is the second most common cause of cancer mortality worldwide. Although chemotherapy is one of the most important treatment options for gastric cancer, and could improve the overall survival rate and quality of live, one significant reason for its failure is multidrug resistance (MDR).

Aim: To study the effect of tumour necrosis factor-related apoptosis-inducing ligand (TRAIL) combined with chemotherapeutic drug cisplatin (DDP) on the expression of multidrug resistance gene 1 (MDR1) in the gastric cancer cell line SGC-7901/VCR.

Material and methods: SGC-7901/VCR cells were cultured with DDP and TRAIL in various concentrations. The apoptosis rate was separately measured by a flow cytometer in DDP (sub-toxic dose) alone, TRAIL $(200 \mu \mathrm{g} / \mathrm{l})$ alone and in a combination of the two. Expression levels of MDR1 mRNA and P-glycoprotein (P-gp) were detected by RT-PCR and ELISA analysis, respectively.

Results: The apoptosis rate in the combination group was significantly higher than that in the other groups $(p<0.05)$. According to the results of RT-PCR and ELISA, the expressions of MDR1 mRNA and P-gp in the combination group were statistically significant different compared with other groups $(p<0.05)$.

Conclusions: The combination of TRAIL with DDP could reverse MDR phenotype in gastric cancer cell line SGC7901/VCR. The mechanism may be involved in the down-regulation of MDR1 mRNA and P-gp, which may play an essential role in overcoming the chemotherapeutic resistance of gastric cancer cells. This study indicates that a combination of chemotherapy and TRAIL may be an effective strategy to treat MDR gastric cancer.
\end{abstract}

\section{Introductions}

Gastric cancer is the fourth most common malignant tumour and second major cause of cancer-associated death worldwide [1, 2]. In China, gastric cancer cases account for almost $40 \%$ of all cancer cases [3]. Chemotherapy remains one of the major treatments for patients who suffer from gastric cancer. However, multidrug resistance (MDR) of tumour cells is the main obstacle to successful treatment, including intrinsic and acquired MDR. At present, the $\mathrm{ABC}$ transporter subfamily B member 1 (MDR1/P-gp), subfamily C member $1 / 2$ (MRP-1/2), subfamily G member 2 (BCRP), and lung resistance-related protein (LRP) have been recognised as the mechanisms mediating MDR to anti-cancer drugs. MDR1, a classic MDR gene, is one of the important causes of tumour resistance. Numerous studies have confirmed that MDR1/P-gp overexpression correlates with
MDR to chemotherapy [4-6]. Therefore, inhibition of P-glycoprotein (P-gp) expression may reverse the MDR phenotype of tumour cells. As a member of the TNF superfamily, tumour necrosis factor-related apoptosis-inducing ligand (TRAIL) could selectively induce apoptosis of tumour cells, without obvious toxicity to normal cells [7]. However, many tumour cells are relatively resistant to TRAIL. The study indicates that decreasing the expression of MDR/p-gp could effectively increase the sensitivity of tumour cells to chemotherapy drugs [8]. To the best of our knowledge, it is not well understood whether the combination of anti-tumour agents and TRAIL could be applied to MDR gastric cancer.
Aim
In the present study we will explore the effective- ness of the combination use of TRAIL and anti-tumour agents on the gastric cancer SGC-7901/VCR cell line. 


\section{Material and methods}

Gastric cancer drug-resistant cell line SCG-7901/VCR was granted by Professor Fan Daiming from the Institute of Digestive Diseases, Xijing Hospital, the Fourth Military Medical University. RPMI 1640 culture fluid and foetal calf serum were purchased from the company Gibco. MDR1 and $\beta$-actin primers were designed and synthesised by Sangon Biotech (Shanghai) Co., Ltd. MTT and DMSO were purchased from the company Sigma; TRIzol and Annexin V-Alexa Fluor 488 test kits were purchased from the company Invitrogen; the AMV first strand cDNA compound reagent kit and PCR amplification reagent kit were both sourced from $M B I$. ELISA test kits were purchased from the company R \& D. TRAIL protein was purchased from the company Peprotech. Vincristine (VCR) was purchased from Shenzhen Main Luck Pharmaceuticals Inc. and cis-platinum injection (cis-diammineplatinum dichloride - DDP) was purchased from Nanjing Pharmaceutical Factory Co., Ltd.

\section{Cell culture}

SGC7901/VCR cells were cultured in RPMI1640 medium containing 10\% foetal bovine serum (FBS), and penicillin $(100 \mathrm{U} / \mathrm{ml})$ and streptomycin $(100 \mathrm{mg} / \mathrm{ml})$ at $37^{\circ} \mathrm{C}$ in an atmosphere of $95 \%$ air and $5 \% \mathrm{CO}_{2}$. The cells were routinely maintained in a medium containing $1.0 \mathrm{mg} / \mathrm{l}$ VCR and incubated in a drug-free medium for 2 weeks before use. The cells were routinely subcultured every 2-3 days and were all from the logarithmic phase of growth.

\section{MTT assay to determine the DDP sensitivity of the cells and to calculate the IC10}

Gastric cancer cells SGC-7901/VCR were plated in a 96-well plate at a density of $5 \times 10^{3}$ cells/well in RPMI 1640 containing 10\% FBS. After the cells had grown adherent to the wall, various concentrations of DDP were added, respectively. Six duplicate wells were set up for each group. The culture medium and the drug were replaced at 24-hour intervals to maintain the drug concentration. Forty-eight hours later, $20 \mu \mathrm{l}$ of $5 \mathrm{mg} / \mathrm{ml}$ MTT was added per well, and the culture was incubated for an additional $4 \mathrm{~h}$. Then, the supernatant was discarded and $150 \mu \mathrm{l} /$ well of dimethyl sulfoxide (DMSO) was added. Spectrometric absorbance at a wavelength of 490 nm (A490) was measured. Each assay was performed in triplicate. The inhibition rate (\%) was calculated according to the following formula: (1- experiment absorbance/ control group absorbance) $\times 100 \%$. And IC10 (concentration at $10 \%$ of inhibitory rate) of DDP was calculated and used as a sub-toxic dosage in the following experiment.
TRAIL preparation and concentration design

TRAIL was melted in $100 \mu$ of double distilled water according to the manufacturer's manual, mixed to $100 \mathrm{mg} / \mathrm{ml}$, kept in $-20^{\circ} \mathrm{C}$ and diluted to the desired concentration with the culture medium on the date of the experiment. Two hundred $\mu \mathrm{g} / \mathrm{l}$ of TRAIL and $0.535 \mathrm{~g} / \mathrm{l}$ of DDP (IC10) were adopted in the test. The IC10 value of DDP was calculated from the MTT assay in our study in accordance with previous studies. The experiment was conducted in four groups: 1) control group: cell + RPMI 1640, 2) DDP group: DDP + cell + RPMI 1640, 3) TRAIL group: $200 \mu \mathrm{g} / \mathrm{I}$ TRAIL + cell + RPMI 1640, and 4) combi-

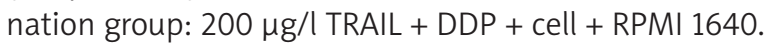

\section{Cell treatment}

Logarithmic phase SGC-7901/VCR cells were taken and inoculated in a culture flask at the rate of $1 \times 10^{5} / \mathrm{ml}$ per well. When the cells had grown adherent to the wall, the culture medium containing the various drug concentrations mentioned previously was replaced at 24-hour intervals to maintain the drug concentration. Cells from the four groups were collected for further experimentation after $48 \mathrm{~h}$.

\section{Flow cytometry analysis}

After the cells were treated by TRAIL or the combination of TRAIL and DDP for $48 \mathrm{~h}$, the cells were harvested and prepared in a cell suspension by adding $100 \mu \mathrm{l}$ of Binding Buffer. Then the samples were incubated in a dark place at $4^{\circ} \mathrm{C}$ for $15 \mathrm{~min}$ after adding $5 \mu \mathrm{l}$ of Annexin $\mathrm{V}$ and PI solutions. Finally, $400 \mu \mathrm{l}$ of Binding Buffer was added to re-suspend the cells. The apoptotic cells were determined by flow cytometry, and the percentage of apoptotic cells was measured with Win MDI software.

\section{Semi-quantitative RT PCR}

RNA isolation

Total RNA was isolated from cells using TRIzol reagent according to the manufacturer's protocol.

Measurement of the total RNA: The resulting A260/280 were both between 1.8 and 2.0, indicating that pure RNA samples were obtained.

Measurement of the RNA integrity: After electrophoresis, a 28S and an $18 \mathrm{~S}$ rRNA strip were clearly seen in the gel under ultraviolet light, suggesting that RNA was completely isolated. The RNA was stored at $-80^{\circ} \mathrm{C}$. $1 \mu \mathrm{g}$ of the total RNA was reverse transcribed into cDNA using an AMV first strand cDNA compound reagent kit. Reaction conditions: reverse at $42^{\circ} \mathrm{C}$ for $1 \mathrm{~h}, 70^{\circ} \mathrm{C}$ for $5 \mathrm{~min}$ and $4^{\circ} \mathrm{C}$ for $10 \mathrm{~min}$, and the obtained cDNA was 


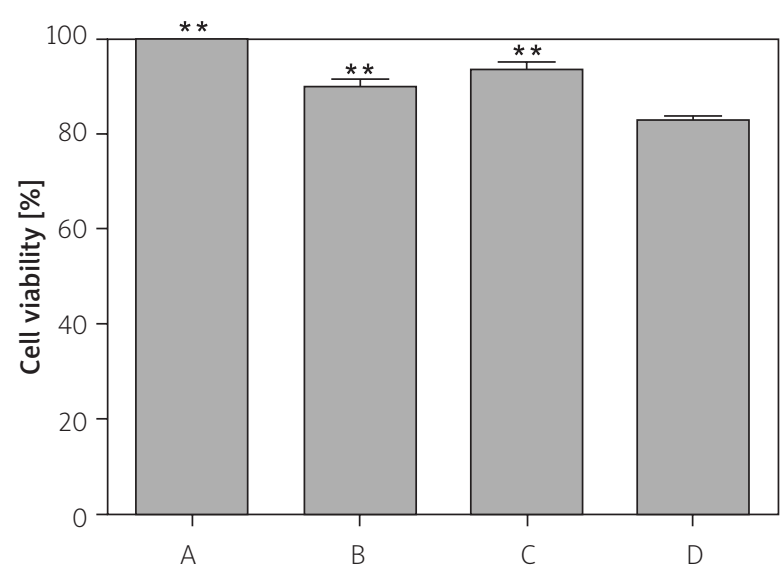

Figure 1. Inhibitory effect on SGC7901/VCR of various groups: A - control group, B - DDP $(0.535 \mathrm{~g} / \mathrm{l}), \mathrm{C}-\mathrm{TRAIL}(200 \mu \mathrm{g} / \mathrm{l})$, and D - TRAIL + DDP. Compared with the combination group: ${ }^{* *} p<0.01$

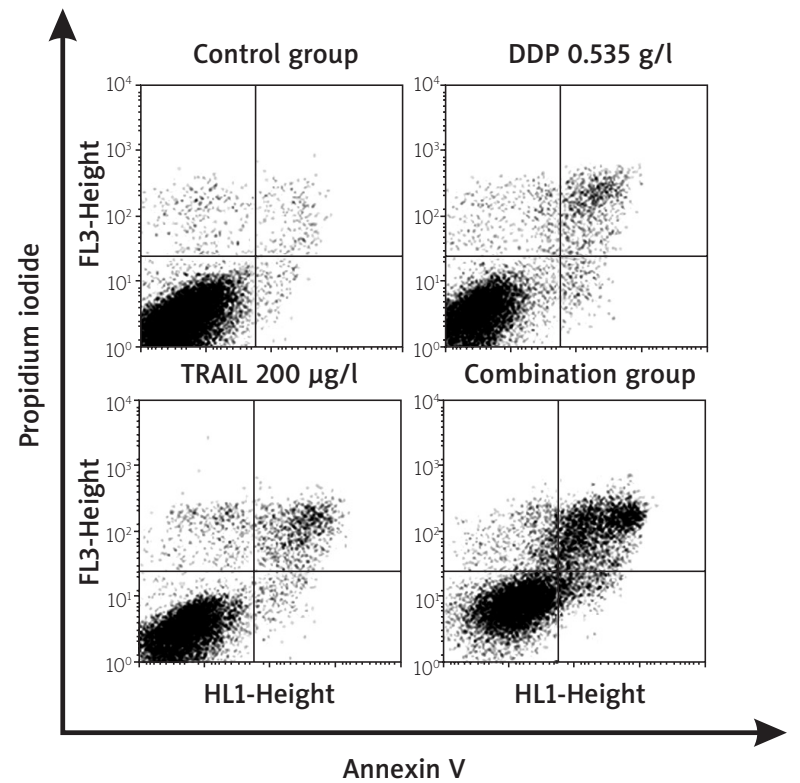

Figure 2. SGC7901/VCR underwent apoptosis after TRAIL + DDP treatment. Cells were cultured for $48 \mathrm{~h}$ with $200 \mu \mathrm{g} / \mathrm{I}$ TRAIL and/or $0.535 \mathrm{~g} / \mathrm{l}$ DDP. Cells were stained with FITC-anti-Annexin V antibody and PI, and subjected to flow cytometric analysis

stored at $-80^{\circ} \mathrm{C} .1 \mu \mathrm{l}$ CDNA was taken for PCR reaction with $\beta$-actin as the internal reference. MDR1 primer: 5'-TGACTACCAGGCTCGCCAATGAT-3' (forward); 5'-TGTGCCACCAAGTAGGCTCCAAA-3' (reverse); and $\beta$-actin primer: 5'-GCCGATCCACACGGAGTACTT-3' (forward); 5'-TTGCCGACAGGATGCAGAA-3' (reverse) were used in
PCR under the following conditions: pre-degeneration at $95^{\circ} \mathrm{C}$ for $5 \mathrm{~min}, 94^{\circ} \mathrm{C}$ for $50 \mathrm{~s}, 57^{\circ} \mathrm{C}$ for $60 \mathrm{~s}$ and $72^{\circ} \mathrm{C}$ for $30 \mathrm{~s}$, amplified 35 cycles, extended $10 \mathrm{~min}$ at $72^{\circ} \mathrm{C}$. The PCR product was analysed using $2 \%$ agarose gel electrophoresis and visualised with ethidium bromide staining.

\section{Detecting expression of P-gp by ELISA method}

Fresh culture medium was replaced when the cells had grown to $50-60 \%$, and the cells were incubated with various concentrations, as mentioned above. The cells of each group were collected after 48-hour intervention, protein lysate was added and centrifuged 15-20 $\mathrm{min}$ at a speed of $12,000 \mathrm{r} / \mathrm{min}$ to remove the cell fragments. Then $500 \mu$ l of supernatant was absorbed and added into the antibody-coated 96-well plate. Subsequently, the light absorbency (A) of each sample at $450 \mathrm{~nm}$ was read from the microplate reader and a standard curve was produced, and then the P-gp in each sample was calculated according to the standard protein provided in the reagent kit. Three duplicate wells were conducted for each concentration and the experiment was repeated three times.

\section{Statistics analysis}

The results obtained were expressed as the mean \pm SD of at least three independent experiments. Statistical analysis was conducted using SPSS 19.0 software. Comparison between the means of the two groups was tested with the $t$-test. Comparison between the means of multiple groups was achieved by single-factor analysis of variance. Value of $p<0.05$ was considered statistically significant in all experiments.

\section{Results}

\section{Cell viability tested by MTT assay}

The inhibitory rate of DDP on SGC7901/VCR cells increases in a dose-dependent manner, and the concentration at IC10 is $0.528 \mathrm{~g} / \mathrm{l}$. The inhibitory rate of TRAIL on the cells increases in a dose-dependent manner within each degree of TRAIL concentration, and $200 \mu \mathrm{g} / \mathrm{l}$ was used in this study. Among the various groups, the survival rate of the cells in the combination group (TRAIL + DDP) was significantly lower than that seen in the single drug groups, and the differences are statistically significant $(p<0.05)$ (Figure 1$)$.

\section{Detection of apoptosis rate by flow cytometry}

Annexin V/PI double staining flow cytometry results show the apoptosis rate in the combination group was $31.13 \pm 1.87 \%$, which was higher than that seen 
in the DDP group $(8.26 \pm 0.98)$ and in the TRAIL group (9.71 \pm 0.53$)$. The results suggest that the combination of TRAIL $(200 \mu \mathrm{g} / \mathrm{l})$ with DDP $(0.535 \mathrm{~g} / \mathrm{l})$ could increase the apoptosis rate when compared with single drug groups, and it is statistically significant $(p<0.05)$ (Figure 2 ).

\section{Effect of the combination of TRAIL and DDP on the expression of MDR1 mRNA}

The TRAIL group and the combination group both show an inhibitory effect on MDR1 expression, but the expression inhibitory effect in the combination group is more obvious. As compared with the control group and the DDP group, MDR1 in the combination group is significantly reduced, and the difference is statistically significant $(p<0.01)$. When compared with the TRAIL group, the difference is statistically significant $(p<0.05)$ (Figure 3).

\section{The influence of single drug and combination use on SGC-7901/VCR cell P-gp expression}

All groups have an inhibitory effect on P-gp expression, among which, the inhibitory effect of the combination group is the most obvious. As compared with the control group and the DDP group, P-gp in the combination group is significantly reduced $(p<0.01)$. When compared with the TRAIL group, the difference is statistically significant $(p<0.05)$ (Figure 4).

\section{Discussion}

Multidrug resistance (MDR) phenotype is the main obstacle in the chemotherapy of cancers, including gastric cancer. One prototypical MDR to anticancer agents is mediated by overexpressed P-gp, which is a $170 \mathrm{kDa}$ transmembrane glycoprotein encoded by the MDR1 gene. Therefore, the role of MDR1/P-gp has been proposed as an important goal in the research aiming to reverse MDR of cancer cells $[9,10]$. The study finds that MDR1/P-gp overexpression is closely related to acquired MDR of cancer cells [11]. Cancer has become the second most common cause of death in China, and although mortality from gastric cancer has decreased substantially over the last few decades, it is still higher than in most developed countries [12]. Chemotherapy is an essential constituent of the comprehensive treatment of gastric cancer, but resistance to anti-cancer agents is the greatest impediment to the chemotherapy of cancer. TRAIL and its agonistic anti-receptor antibodies have gained much attention due to their specific anti-tumour action without toxic side effects, and they look to be promising anti-tumour agents [13]. Howev-

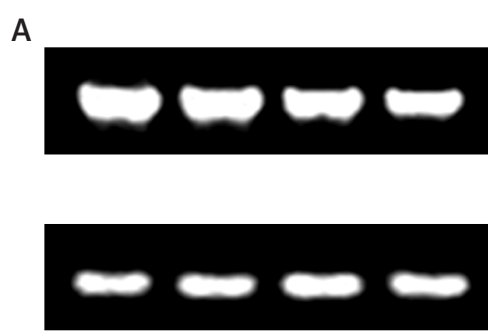

MDR1

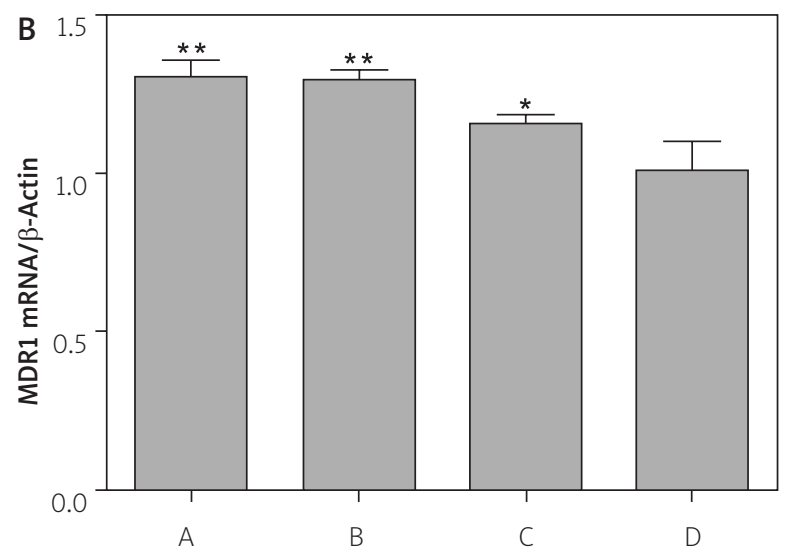

Figure 3. Expression of MDR1 mRNA of SGC7901/ VCR in each group: $\mathbf{A}$ - control group, $\mathbf{B}-$ DDP (0.535 g/l), C - TRAIL $(200 \mu \mathrm{g} / \mathrm{l})$, and D - TRAIL + DDP. Compared with the combination group: ${ }^{*} p<0.05,{ }^{* *} p<0.01$

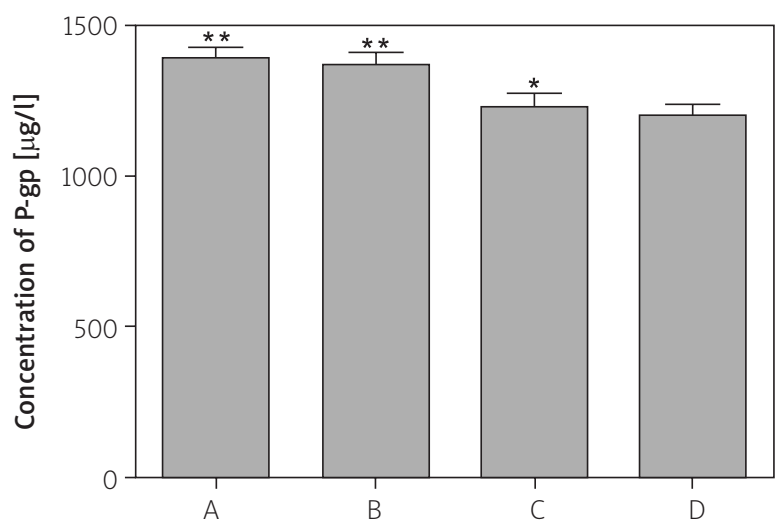

Figure 4. Expression of P-gp of SGC7901/VCR in each group: A - control group, B - DDP (0.535 g/l), C - TRAIL $(200 \mu \mathrm{g} / \mathrm{l})$, and D - TRAIL + DDP. Compared with the combination group: ${ }^{*} p<0.05,{ }^{* *} p<0.01$

er, a variety of tumour cells are relatively resistant to TRAIL. Numerous research projects have demonstrated that anti-tumour agents could sensitise tumour cells to TRAIL-induced apoptosis, indicating that a combination of anti-tumour agents and TRAIL may be an effective 
strategy to kill TRAIL-resistant tumour cells. Additionally, this sensitisation is accompanied by up-regulation of death receptor 5 (DR5) expression and caspase activation $[14,15]$. There is research showing that TRAIL can significantly potentiate the cytotoxicity of anti-tumour drugs, which are P-gp substrates in MDR cells, indicating that TRAIL has a reversal effect on MDR phenotypes [16]. However, no report has yet been published as to whether the combination strategy (TRAIL and anti-tumour agents) reverses the MDR of gastric cancer cells by decreasing the expression of MDR1 and P-gp. The previous study by our research group found that TRAIL could inhibit the expression of MDR1, LRP and GST- $\pi$ mRNA in a dosage-dependence manner within a certain level of TRAIL concentration [17]. In the present study, the results of MTT assay and flow cytometry show that TRAIL has little influence on the activity of SGC-7901/ VCR cells. DDP (sub-toxic dose) also has little influence on the activity of the cells. However, the combination of TRAIL and DDP could inhibit the survival rate and significantly increase the apoptosis rate of SGC7901/VCR cells, and it shows a synergy effect, which is consistent with the conclusions of previous studies $[18,19]$. In the present study, we found that either TRAIL or the combination of TRAIL and DDP could inhibit the expression of MDR1 and P-gp in SGC7901/VCR cells, and when compared with the TRAIL group, the difference is significant. The mechanism of the synergy effect remains unclear, which may be related to the following factors: 1) The early studies reported that a combination of TRAIL and chemotherapy drugs could induce expression of TRAIL protein and TRAIL receptors, inducing TRAIL-induced apoptosis [20]. 2) TRAIL and DDP can induce apoptosis and can be intersected in their apoptosis chains, which can be expressed as cascade amplification of apoptosis. 3) The study finds that TRAIL may increase the accumulation of chemotherapy drugs in tumour cells by decreasing the expression of P-gp, and increase the sensitivity of tumour cells to chemotherapy drugs [21].

\section{Conclusions}

Our research shows the following: a combination of TRAIL and a small dose of (sub-toxic) DDP could significantly decrease the survival rate and increase the apoptosis rate of SGC-7901/VCR cells. The mechanism may involve reversing the MDR phenotype by decreasing the expression of MDR1 mRNA and P-gp. In this study, DDP is at a sub-toxic dose, which also reduces the cell toxicity effects and improves the compliance of patients, and the combination strategy may become a promising method to overcome MDR encountered in gastric cancer treatment. However, the mechanism involved in decreasing the expression of MDR1 mRNA and P-gp by the combination strategy in this study is not well understood, so further study is requried.

\section{Acknowledgments}

We would like to thank Prof. Daiming Fan, Director of the Institute of Digestive Diseases, Xijing Hosiptal, Fourth Military Medical University, for donating the human gastric cancer drug-resistant cell line SGC-7901/VCR.

\section{References}

1. Zuk K, Peczek L, Stec-Michalska K, et al. Family history of gastric cancer correlates with decreased expression of HINT1 tumor suppressor gene in gastric mucosa of dyspeptic patients. Oncol Lett 2012; 3: 219-23.

2. Anderson WF, Camargo MC, Fraumeni JF, et al. Age-specific trends in incidence of noncardia gastric cancer in US adults. JAMA 2010; 303: 1723-8.

3. Yang L. Incidence and mortality of gastric cancer in China. World J Gastroenterol 2006; 12: 17-20.

4. Sheng X, Zhang L, Tong N, et al. MDR1 C3435T polymorphism and cancer risk: a meta-analysis based on 39 case-control studies. Mol Biol Rep 2012; 39: 7237-49.

5. Lee SW, Lee YL, Lee YJ, et al. Enhanced antitumor effects by combination gene therapy using MDR1 gene shRNA and HSV1tk in a xenograft mouse model. Cancer Lett 2010; 291: 83-9.

6. Lin X, Zhang X, Wang Q, et al. Perifosine downregulates MDR1 gene expression and reverses multidrug-resistant phenotype by inhibiting PI3K/Akt/NF-kB signaling pathway in a human breast cancer cell line. Neoplasma 2012; 59: 248-56.

7. WU GS. TRAIL as a target in anti-cancer therapy. Cancer Lett 2009; 285: 1-5.

8. Kwon HC, Roh MS, Oh SY, et al. Prognostic value of expression of ERCC1, thymidylate synthase, and glutathione S-transferase P1 for 5-fluorouracil/oxaliplatin chemotherapy in advanced gastric cancer. Ann Oncol 2007; 18: 504-9.

9. Li X, Li JP, Yuan HY, et al. Recent advances in P-glycoprotein-mediated multidrug resistance reversal mechanisms. Methods Find Exp Clin Pharmacol 2007; 29: 607-17.

10. Schaefer U, Voloshanenko O, Willen D, et al. TRAIL: a multifunctional cytokine. Front Biosci 2007; 12: 3813-24.

11. Takakuwa O, Oguri T, Ozasa H, et al. Over-expression of MDR1 in amrubicinol-resistant lung cancer cells. Cancer Chemother Pharmacol 2011; 68: 669-76.

12. Guo P, Huang ZL, Yu P, et al. Trends in cancer mortality in China: an update. Ann Oncol 2012; 23: 2755-62.

13. Testa U. TRAIL/TRAIL-R in hematologic malignancies. J Cell Biochem 2010; 110: 21-34.

14. Guo LG, Fan L, Pang ZQ, et al. TRAIL and doxorubicin combination enhances anti-glioblastoma effect based on passive tumor targeting of liposomes. J Control Release 2011; 154: 93-102.

15. Jacquemin G, Shirley S, Micheau O. Combining naturally occurring polyphenols with TNF-related apoptosis-inducing ligand: a promising approach to kill resistant cancer cells? Cell Mol Life Sci 2010; 67: 3115-30.

16. Younes A, Kadin ME. Emerging applications of the tumor necrosis factor family of ligands and receptors in cancer therapy. J Clin Oncol 2003; 21: 3526-34. 
17. Zhang KG, Qin CY, Wang HQ, et al. The effect of TRAIL on the expression of multidrug resistant genes MDR1, LRP and GST- $\pi$ in drug-resistant gastric cancer cell SGC7901/VCR. Hepatogastroenterology 2012; 59: 2672-6.

18. Dolloff NG, Mayes PA, Hart LS, et al. Off-target lapatinib activity sensitizes colon cancer cells through TRAIL death receptor up-regulation. Sci Transl Med 2011; 3: 86ra50.

19. Moon DO, Kang CH, Kang SH, et al. Capsaicin sensitizes TRAIL-induced apoptosis through Sp1-mediated DR5 up-regulation: involvement of Ca2+ influx. Toxicol Appl Pharmacol 2012; 259: 87-95.

20. Lacour S, Hammann A, Wotawa A, et al. Anticancer agents sensitize tumor cells to tumor necrosis factor-related apoptosis-inducing ligand mediated caspase- 8 activation and apoptosis. Cancer Res 2001; 61: 1645-51.

21. Wu Y, Fan Y, Xue B, et al. Human glutathione S-transferase P1-1 interacts with TRAF2 and regulates TRAF2-ASK1 signals. Oncogene 2006; 25: 5787-800.

Received: 11.10.2013

Accepted: 17.01 .2014 